TRANSACTIONS OF THE

AMERICAN MATHEMATICAL SOCIETY

Volume 359, Number 1, January 2007, Pages 19-32

S 0002-9947(06)04198-5

Article electronically published on August 15, 2006

\title{
DIFFERENTIABILITY OF QUASI-CONFORMAL MAPS ON THE JUNGLE GYM
}

\author{
ZSUZSANNA GÖNYE
}

\begin{abstract}
We obtain a result on the quasi-conformal self-maps of jungle gyms, a divergence-type group. If the dilatation is compactly supported, then the induced map on the boundary of the covering disc $\mathbb{D}$ is differentiable with non-zero derivative on a set of Hausdorff dimension 1 .

As one of the corollaries, we show that there are quasi-symmetric homeomorphisms over divergence-type groups such that for all sets $E$ the Hausdorff dimension of $E$ and $f\left(E^{c}\right)$ cannot both be less than 1. This shows an important difference between finitely generated and divergence-type groups.
\end{abstract}

\section{INTRODUCTION}

Consider a discrete Möbius group $G$ acting on the unit disk $\mathbb{B}^{n}, n \geq 2$. Let $f$ be a homeomorphism of $S^{n-1}=\partial \mathbb{B}^{n}$ which conjugates $G$ to another Möbius group $G^{\prime}$. The properties of this conjugating map $f$ have been studied extensively over the years.

According to the famous result of Mostow [11, if $G$ is cocompact and $n \geq 3$, then $f$ is Möbius, and therefore the quotient manifolds $\mathbb{B}^{n} / G$ and $\mathbb{B}^{n} / G^{\prime}$ are conformally equivalent. Mostow later extended his theorem to the case of $n=2$ and gained the dichotomy, the Mostow rigidity theorem: $f$ is either completely singular with respect to the $(n-1)$-dimensional Lebesgue measure on $S^{n-1}$, or it is a Möbius transformation [12]. In [1] Agard showed that divergence-type groups also have the Mostow rigidity property. For Fuchsian groups this latter result is sharp, as was shown by Astala and Zinsmeister in 22. In [15] Tukia gave further conditions in which $f$ must be Möbius: if $f$ is differentiable with non-vanishing Jacobian at a radial point of $G$, then $f$ is a Möbius transformation unless there is a point fixed by every element of $G$.

Let us approach these results from a slightly different point of view. Divergenttype Fuchsian groups have the Mostow rigidity property, so if $f$ is a conjugating but not a Möbius map from $G$ to $G^{\prime}$, then it must be completely singular. Instead of Lebesgue measure we can investigate the Hausdorff measure of these sets. The paper [5] by Bishop and Steger states the following result: for finitely generated Fuchsian groups, there is a set $E$ so that

$$
\max \left(\operatorname{dim}_{\mathrm{H}}(E), \operatorname{dim}_{\mathrm{H}}\left(f\left(E^{c}\right)\right)\right)<1,
$$

Received by the editors September 9, 2004.

2000 Mathematics Subject Classification. Primary 30C62, 28A78; Secondary 30F35.

Key words and phrases. Fuchsian group, Kleinian group, quasi-conformal map, jungle gym, Hausdorff dimension.

(C)2006 American Mathematical Society Reverts to public domain 28 years from publication 


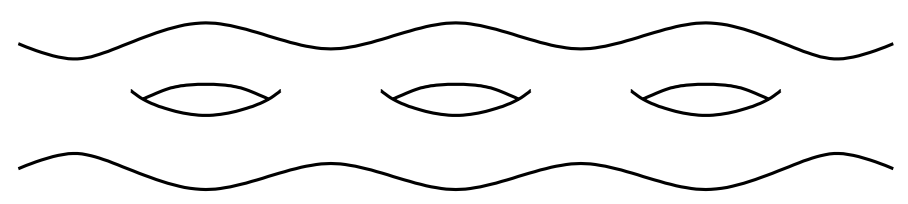

FiguRE 1. 1-dimensional jungle gym

where $\operatorname{dim}_{\mathrm{H}}$ denotes the Hausdorff dimension of the set, and $f$ is a conjugating homeomorphism of $\mathbb{B}$.

In this paper we will show that this above result is not true for divergence-type groups. Namely, we will construct a conjugating map $f$ between covering groups of two jungle gyms for which

$$
\max \left(\operatorname{dim}_{\mathrm{H}}(E), \operatorname{dim}_{\mathrm{H}}\left(f\left(E^{c}\right)\right)\right)=1
$$

for all $E \subset S^{1}$.

Suppose $G$ is a Fuchsian group covering of the '1-dimensional jungle gym', pictured in Figure 1. Consider a quasi-conformal deformation $f$ of this surface by using a dilatation in a ball $U$ with compact closure on the 'jungle gym', i.e. on the quotient Riemann surface $\mathbb{D} / G$. We can lift this map $f$ to the universal covering space, to the hyperbolic disc $\mathbb{D}$. The lifted map $F: \mathbb{D} \rightarrow \mathbb{D}$ is a quasi-conformal self-map of the hyperbolic disc, it has the same complex dilatation as $f$, and the dilatation is supported in the lifts of the ball $U$, i.e. in a union of hyperbolic balls in $\mathbb{D}$. Moreover, any quasi-conformal self-mapping of a disc, in particular this map $F$, admits a homeomorphic extension to the boundary. Outside the unit ball $F$ is defined by the reflection across the boundary of the unit ball. Our first theorem states that this extended map, that we also call $F$, is differentiable with a non-zero derivative on a set of Hausdorff dimension of 1 .

Theorem 1.1. Let $f$ be a quasi-conformal self-map of the jungle gym so that the dilatation of $f$ is compactly supported, and let $F$ be its lifted map to the hyperbolic disc extended to the boundary of the disc. Then $F$ is differentiable with non-zero derivative at the deep points, and the Hausdorff dimension of these points is 1.

The outline of the proof is the following: to show that the function $F$ is differentiable with non-zero derivative at the deep points, we are going to use a theorem by O. Lehto from [7. Then, we would like to use Theorem [5.1 from [6] to prove that the set of deep points has full dimension. Although the proof relies on the existence of a Green's function on the quotient manifold (Lemma 5.2 of this paper), in the special case of the jungle gym we can still make the theorem work, as follows. Sullivan in 14 showed that on manifolds like the jungle gym, there is no positive non-constant superharmonic function, so there is no positive non-constant harmonic function either. But we can construct a harmonic function on each half of the jungle gym separately. Cut the jungle gym into two quasi-cylinders with a curve through the point $z_{0}$. Then on each quasi-cylinder, $M_{i}$, we can construct a positive harmonic function, $h_{i}$, so that

$$
\frac{1}{c} \operatorname{dist}\left(x, \partial M_{i}\right) \leq h_{i}(x) \leq c \operatorname{dist}\left(x, \partial M_{i}\right)
$$

with some constant $c<\infty$. For these harmonic functions we can apply Theorem 5.1 . which shows that the dimension of deep points has full dimension. 
As a result, our main corollary states that the Bishop-Steger theorem fails for some divergence-type groups.

Corollary 1.2. Let $F$ be a quasi-symmetric conjugating map of two jungle gyms whose dilatation has compact support. Then $F$ is differentiable with non-zero derivative on a set of Hausdorff dimension 1, and the image of this set also has dimension 1. Moreover, $\max \left(\operatorname{dim}_{\mathrm{H}}(E), \operatorname{dim}_{\mathrm{H}}\left(F\left(E^{c}\right)\right)\right)=1$ for all $E \subset S^{1}$.

\section{Definitions and notations}

2.1. Quasi-conformal maps. There is more than one way to define quasiconformal maps. The definition we give here is called the analytic definition. Another equivalent definition, the so-called geometric definition, can be found in several books, for example in [9] or [10].

Let $f$ be a diffeomorphism between domains $A$ and $B$ of the extended complex plane, i.e. a homeomorphism which with its inverse is continuously differentiable. We also assume that $f$ is a sense-preserving map, i.e. $J(f)=|\partial f|^{2}-|\bar{\partial} f|^{2}>0$. The dilatation quotient is defined by

$$
D_{f}=\frac{\max _{\alpha}\left|\partial_{\alpha} f\right|}{\min _{\alpha}\left|\partial_{\alpha} f\right|}=\frac{|\partial f|+|\bar{\partial} f|}{|\partial f|-|\bar{\partial} f|},
$$

where

$$
\partial_{\alpha} f=\lim _{r \rightarrow 0} \frac{f\left(z+r e^{i \alpha}\right)-f(z)}{r e^{i \alpha}}=\partial f+\bar{\partial} f e^{-2 i \alpha}
$$

is the derivative of $f$ in direction $\alpha$. We call a sense-preserving diffeomorphism $f$ $K$-quasi-conformal if $D_{f}(z) \leq K$ holds everywhere.

Alternatively, we can define the function

$$
\mu=\frac{\bar{\partial} f}{\partial f}
$$

which is called the complex dilatation of $f$. This is a continuous function and $|\mu(z)|<1$. A sense-preserving diffeomorphism $f: A \rightarrow B$ is quasi-conformal if $\sup _{z \in A}|\mu(z)|<1$. In particular, $f$ is $K$-quasi-conformal $(K \geq 1)$ if

$$
|\mu(z)| \leq \frac{K-1}{K+1}<1
$$

for all $z \in A$.

Here we mention a result from [10] on the extension of a quasi-conformal map, that we will use later. A quasi-conformal map $f$ of a Jordan domain $A$ onto another Jordan domain $B$ can always be extended to a homeomorphism from the closure of $A$ to the closure of $B$. In particular, a quasi-conformal self-map on a disc can be extended to the boundary of the disc homeomorphically.

2.2. Deformation of Riemann surfaces. A homeomorphism $F: S_{1} \rightarrow S_{2}$ between the Riemann surfaces $S_{1}$ (with atlas $H_{1}$ ) and $S_{2}$ (with atlas $H_{2}$ ) is called $K$-quasi-conformal if $w=h_{2} \circ F \circ h_{1}^{-1}$ is K-quasi-conformal on its domain for all $h_{i} \in H_{i}$, and $\mu=\frac{\bar{\partial} w}{\partial w}$ exists almost everywhere in the domain of $w$. We call this $\mu$ the complex dilatation of $F$.

Let $S_{i}$ be a Riemann surface and $\left(D, \Pi_{i}\right)$ a universal covering space of $S_{i}$, and $G_{i}$ the cover transformation group, i.e. $D / G_{i}$ is equivalent to $S_{i}$. Suppose $f$ is a 


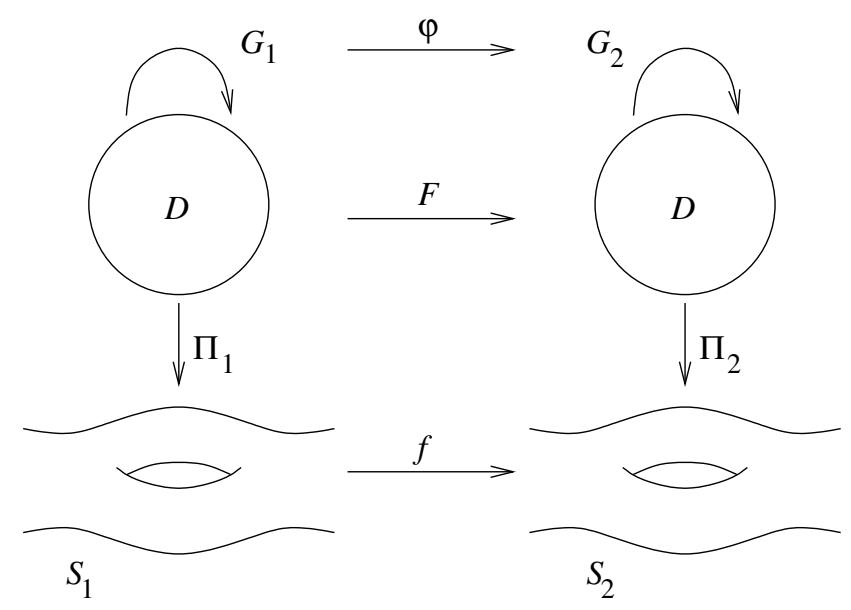

FiguRE 2. Deformation of Riemann surfaces

continuous map from $S_{1}$ into $S_{2}$ (see Figure 2). Then $f$ induces a continuous map $F: D \rightarrow D$ so that

$$
f \circ \Pi_{1}=\Pi_{2} \circ F \text {. }
$$

If $f$ is a homeomorphism, then so is the lifted map $F$; if $f$ is conformal, then $F$ is conformal [8].

The map $f$ also induces a map $\phi: G_{1} \rightarrow G_{2}$. Take $g_{1} \in G_{1}$, then $\Pi_{2} \circ F \circ$ $g_{1}=f \circ \Pi_{1} \circ g_{1}=f \circ \Pi_{1}=\Pi_{2} \circ F$. Therefore for every $z \in D$ there is a transformation $g_{2} \in G_{2}$ such that $\left(F \circ g_{1}\right)(z)=\left(g_{2} \circ F\right)(z)$. Define $\phi\left(g_{1}\right)=g_{2}$. If $f$ is continuous, then this map $\phi$ is a well-defined homomorphism and bijective if $F$ is bijective. Conversely, a continuous map $F: D \rightarrow D$ can be projected to a mapping $f: S_{1} \rightarrow S_{2}$ if and only if $G_{2} F=F G_{1}$.

2.3. Hausdorff dimension. Suppose $\phi$ is an increasing, continuous function from $[0, \infty)$ to itself such that $\phi(0)=0$. For a given set $E$ we define the Hausdorff content as

$$
\mathcal{H}_{\infty}^{\phi}(E)=\inf \left\{\sum \phi\left(r_{j}\right): E \subset \cup_{j} D\left(x_{j}, r_{j}\right)\right\}
$$

In particular, if $\phi(t)=t^{\alpha}$ we denote $\mathcal{H}_{\infty}^{\phi}$ by $\mathcal{H}^{\alpha}$. The Hausdorff dimension of this set $E$ is

$$
\operatorname{dim}_{\mathrm{H}}(E)=\inf \left\{\alpha: \mathcal{H}^{\alpha}(E)=0\right\}
$$

For more details on Hausdorff dimension see [4].

\section{Rigidity OF MÖBIUS GROUPS}

3.1. Möbius groups. Let $G_{1}$ and $G_{2}$ be two groups of Möbius transformations of $\overline{\mathbb{R}}^{n}$, and let $A_{i}$ be a $G_{i}$-invariant subset of $\overline{\mathbb{R}}^{n}$, i.e. $g\left(A_{i}\right)=A_{i}$ for all $g \in G_{i}$. We say that a map $f: A_{1} \rightarrow A_{2}$ is $G_{1}$-compatible if there is a homomorphism $\phi: G_{1} \rightarrow G_{2}$ such that

$$
f \circ g(x)=\phi(g) \circ f(x)
$$

for all $g \in G_{1}$ and $x \in A_{1}$. In this case we call $\phi$ the induced homomorphism by $f$. 
A map $f: U \rightarrow \overline{\mathbb{R}}^{n}, U \subset \overline{\mathbb{R}}^{n}$, is differentiable at a point $x \in \mathbb{R}^{n}$ if there is an affine map $\alpha$ of $\mathbb{R}^{n}$ so that

$$
\frac{|f(y)-\alpha(y)|}{|y-x|} \rightarrow 0 \quad \text { as } y \rightarrow x \text { in } U,
$$

where $\overline{\mathbb{R}}^{n}$ denotes $\mathbb{R}^{n} \cup \infty$. If the map $\alpha$ can be chosen to be an affine homeomorphism, then $f$ is differentiable with a non-vanishing Jacobian at $x$.

A point $x \in \overline{\mathbb{R}}^{n}$ is called a radial point of the group $G$ (point of approximation or conical point) if there exists a sequence of different $g_{i} \in G$ so that for a given $z \in \mathbb{H}^{n+1}=\left\{\left(x_{1}, x_{2}, \ldots, x_{n}, y\right) \in \mathbb{R}^{n+1}: y>0\right\}$ and for a hyperbolic line $L$ terminating at the point $x$, the hyperbolic distances $\operatorname{dist}\left(g_{i}(z), L\right)$ are all bounded, and $\lim _{i \rightarrow \infty} g_{i}(z)=x$ in $\overline{\mathbb{H}}^{n+1}=\mathbb{H}^{n+1} \cup \infty$. On the quotient manifold this means that there is a geodesic ray returning to a compact set infinitely often.

Tukia showed ([15], Theorem A) that if $G=G_{1}=G_{2}$ is a group of Möbius transformations of $\overline{\mathbb{R}}^{n}$ and $f: \overline{\mathbb{R}}^{n} \rightarrow \overline{\mathbb{R}}^{n}$ is a $G$-compatible map which is differentiable with a non-vanishing Jacobian at a radial point of $G$, then it is a Möbius transformation unless there is a point $z \in \overline{\mathbb{R}}^{n}$ fixed by every element $g \in G$. If there is such a point $z$ fixed by every element of $G$, then there are two Möbius transformations $h$ and $h^{\prime}$ so that $\left.h^{\prime} f h\right|_{\mathbb{R}^{n}}$ is an affine homeomorphism.

In the same paper, Tukia also showed ([15, Theorem D) a similar result for maps defined on a smaller $G$-compatible set, for example if the function is defined on the limit set of the group. The theorem says that if $A \subseteq \overline{\mathbb{R}}^{n}$ is a $G$-invariant set containing at least three points and if $f: A \rightarrow \overline{\mathbb{R}}^{n}$ is a $G$-compatible map of $\overline{\mathbb{R}}^{n}$ which is differentiable with a non-vanishing Jacobian at a radial point $x$ of $G$, then $f$ is an affine map of $\overline{\mathbb{R}}^{n}$, up to composition with Möbius transformations. Moreover, if $A$ is a $k$-sphere for some $k \leq n$ (i.e. $A$ is the image of $\overline{\mathbb{R}}^{k}$ under a Möbius transformation of $\overline{\mathbb{R}}^{n}$ ) and there is no point fixed by every element $g$ of $G$, then $f$ is a Möbius transformation. In the theorem it is essential that $x$ is a radial point ([15, Theorem D2). In the second part it is necessary that there is no common fixed point in $G$.

3.2. Divergence-type groups. A discrete group $G$ fixing the $n$-dimensional unit ball $\mathbb{B}$ is called divergence type if the series

$$
\sum_{g \in G}(1-|g(0)|)^{n-1}
$$

diverges. The convergence of the series above is necessary and sufficient for the existence of a Green's function on the quotient manifold $\mathbb{B} / G$. A discrete group $G$ has finite covolume if it has a fundamental region in $\mathbb{B}$ of finite hyperbolic volume. If the group $G$ preserves $\mathbb{B}$ and has finite covolume, then $G$ is of divergence type [13.

Let $G_{1}$ and $G_{2}$ be two discrete Möbius groups acting on $\overline{\mathbb{R}}^{n}, n \geq 2$, such that $\mathbb{H}^{n+1} / G_{i}$ has finite hyperbolic volume, and let $\phi: G_{1} \rightarrow G_{2}$ be an isomorphism. Then there is a quasi-conformal map $f: \overline{\mathbb{R}}^{n} \rightarrow \overline{\mathbb{R}}^{n}$ inducing $\phi$, and this map $f$ is a Möbius transformation. More generally, if $G$ is a discrete Möbius group of divergence type, and $f: \overline{\mathbb{R}}^{n} \rightarrow \overline{\mathbb{R}}^{n}, n \geq 2$, is a quasi-conformal and $G$-compatible map, then $f$ is a Möbius transformation [15. Agard's result for quasi-conformal deformations: let $G$ be a discrete group of Möbius transformations in $\overline{\mathbb{R}}^{n}$ and suppose it is of divergence type. Let $f: \mathbb{R}^{n} \rightarrow \mathbb{R}^{n}$ be a quasi-conformal map 
such that $f g f^{-1}$ is a Möbius transformation for all $g \in G$. Then if $n \geq 2, f$ is a Möbius transformation, and if $n=1$, then $f$ is either a Möbius transformation or it is singular [1. In dimension one, instead of quasi-conformal we mean quasisymmetric, and the map $f: \overline{\mathbb{R}}^{1} \rightarrow \overline{\mathbb{R}}^{1}$ can be very irregular. These results are often quoted as follows. Let $G$ be a discrete group of Möbius transformations on $\mathbb{H}^{n+1}$ (or on $\mathbb{B}^{n+1}$ ), $n \geq 1$. We say that $G$ has the Mostow rigidity property if for each homeomorphism $f: \overline{\mathbb{R}}^{n} \rightarrow \overline{\mathbb{R}}^{n}$ (or $S^{n}$ ) with $f \circ G \circ f^{-1}$ a Möbius group, it holds that either $f$ is a Möbius transformation itself or completely singular. Therefore the divergence groups have the Mostow rigidity property for all $n \geq 1$. A stronger result for Fuchsian groups is due to Astala and Zinsmeister [2]: a Fuchsian group has the Mostow rigidity property if and only if it is of divergence type.

For finitely generated groups Bishop and Steger showed (see [5]) that if $f$ is not a Möbius map, then there is a set $E$ such that both $\operatorname{dim}(E)$ and $\operatorname{dim}\left(f\left(E^{c}\right)\right)$ are less than 1. In this paper we will show that such a conclusion is not valid for all divergence type groups, in particular we construct a map over the jungle gym such that $\max \left(\left(\operatorname{dim}(E), \operatorname{dim}\left(f\left(E^{c}\right)\right)\right)=1\right.$ for all sets $E$.

\section{Differentiable points on the Jungle Gym}

Consider the original assumptions that $G$ is a Fuchsian group covering the '1dimensional jungle gym', and let $f$ be a quasi-conformal deformation $f$ of this surface by using a dilatation in a ball $U$ with compact closure on the 'jungle gym'. Lift this map $f$ to the universal covering space (see Figure 21), to the hyperbolic disc $\mathbb{D}$. The lifted map $F: \mathbb{D} \rightarrow \mathbb{D}$ is a quasi-conformal self-map of the hyperbolic disc. It has the same complex dilatation as $f$, and the dilatation is supported in the lifts of the ball $U$, i.e. in a union of hyperbolic balls in $\mathbb{D}$. As we mentioned in Section 2.1 a well-known result is that this quasi-conformal self-mapping $F$ of a disc admits a homeomorphic extension to the boundary. We would like to show that this extended map, which we also name $F$, is differentiable with a non-zero derivative on a set of Hausdorff dimension 1.

We use the following theorem due to Lehto [7 on differentiability of quasiconformal maps to show that the corresponding boundary map on the circle is differentiable with non-zero derivative at the deep points.

Theorem 4.1. In a domain $D$, let $\mu(z)$ be measurable with $\sup |\mu(z)|=k<1$, and let $w=w(z)$ be a quasi-conformal mapping whose complex dilatation is equal to $\mu(z)$ a.e. If

$$
I\left(z_{0}\right)=\iint_{D} \frac{\left|\mu(z)-\mu\left(z_{0}\right)\right|}{\left|z-z_{0}\right|^{2}} d \sigma<\infty, z_{0} \in D,
$$

then at $z=z_{0}, w(z)$ is totally differentiable, $J(z)>0$, and $w(z)$ has the complex dilatation $\mu\left(z_{0}\right)$.

First, we will show that the integral in Lehto's theorem is finite over a small Euclidean ball around every deep point. Fix a point $z_{0}$ inside $U$, where $U$ denotes the compact support of the dilatation, and consider a geodesic ray $\gamma(t)$ corresponding to this deep point and starting at the point $z_{0}$. By the definition of deep points this means

$$
1 \geq \frac{\operatorname{dist}\left(\gamma(t), z_{0}\right)}{t} \geq \delta>0
$$




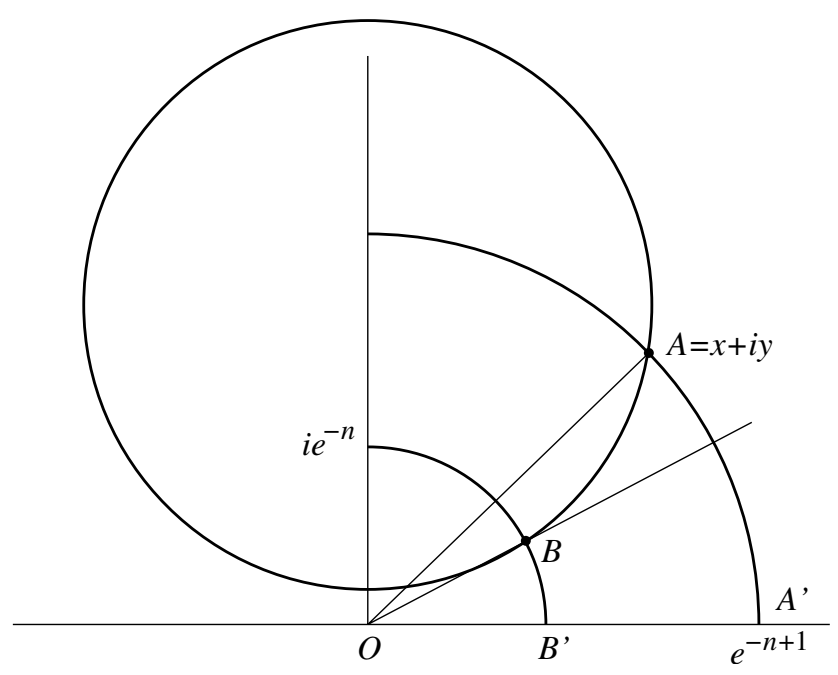

FiguRE 3. Estimate for the integral

for all $t$, i.e. $\operatorname{dist}(\gamma(t), U)>\delta t-D$ for all $t>\frac{D}{\delta}$, where $D$ denotes the diameter of $U$. Because of this inequality there is a region around this geodesic that none of the lifted preimages of $U$ can hit. Call this set $H_{1}$ and its symmetric image over the boundary $H_{2}$. We know that $\mu=0$ in the two regions $H_{1}$ and $H_{2}$ touching the ideal boundary at the deep limit point $x_{0}$. Therefore it will be enough to show that the integral $I\left(x_{0}\right)$ in Lehto's theorem is finite in a neighborhood of $x_{0}$ outside these regions $H_{1}$ and $H_{2}$.

Let $A_{n}$ denote the annulus with radii $e^{-n+1}$ and $e^{-n}$ about the point $x_{0}$. We will use the upper half plane model as the universal covering space for the surface. We may also assume that $x_{0}=0$ and the initial point of the geodesic ray is $\Pi^{-1}\left(z_{0}\right)=i$. With the following two lemmas we shall show that the integral in Lehto's theorem over the set $\bigcup_{n}\left(A_{n} \backslash H_{j}\right)$ is finite, $j=1,2$.

Lemma 4.2. $\iint_{A} d \sigma \leq c e^{-2 n} e^{-\delta n}$.

$$
A_{n} \backslash H_{1}
$$

Proof. Since $\operatorname{dist}(\gamma(t), U)>\delta t-D$ for all $t>t_{0}$, in particular for $t=n$, the set $U$ cannot intersect with the hyperbolic ball of radius $\delta n-D$ around the point $i e^{-n}$. Estimate the area of $A_{n} \backslash H_{1}$ by the area of the wedge shown in Figure 3 For this estimation we need to find the angle $\alpha=A O A^{\prime} \angle$ of the wedge. Since $0 \leq \alpha \leq \frac{\pi}{2}$, we know that $\frac{2}{\pi} \alpha \leq \sin \alpha$, so it is enough to estimate $\sin \alpha=\frac{y}{e^{-n+1}}$.

The point $A=x+i y$ is the intersection of the hyperbolic ball with the Euclidean ball, so

$$
\begin{aligned}
x^{2}+\left(y-e^{-n} \cosh (\delta n-D)\right)^{2} & =\left(e^{-n} \sinh (\delta n-D)\right)^{2}, \\
x^{2}+y^{2} & =e^{-2 n+2} .
\end{aligned}
$$

Eliminating $x^{2}+y^{2}$, we get that

$$
e^{-2 n+2}-2 y e^{-n} \cosh (\delta n-D)+e^{-2 n} \cosh ^{2}(\delta n-D)=e^{-2 n} \sinh ^{2}(\delta n-D),
$$


and rearranging this equation we have that

$$
e^{-n}\left(1+e^{2}\right)=2 y \cosh (\delta n-D) .
$$

Therefore

$$
\begin{aligned}
\sin \alpha & =\frac{y}{e^{-n+1}} \\
& =\frac{1+e^{2}}{2 e \cosh (\delta n-D)} \\
& \leq \frac{1+e^{2}}{2 e} \frac{1}{e^{\delta n-D}} \\
& =\frac{\left(e^{2}+1\right) e^{D-1}}{2} e^{-\delta n} .
\end{aligned}
$$

We should also justify that the picture above is correct, i.e. the wedge does cover the whole set $A_{n} \backslash H_{1}$. For this we have to show that $A O A^{\prime} \angle$ is bigger than $B O B^{\prime} \angle$, or equivalently $\sin \alpha=\sin \left(A O A^{\prime} \angle\right)>\sin \left(B O B^{\prime} \angle\right)=\sin \beta$. We can calculate $\sin \beta$ similarly as we did for $\sin \alpha$. Point $B$ is the intersection of the same hyperbolic ball and the Euclidean ball of radius $e^{-n}$, so

$$
\begin{aligned}
x^{2}+\left(y-e^{-n} \cosh (\delta n-D)\right)^{2} & =\left(e^{-n} \sinh (\delta n-D)\right)^{2}, \\
x^{2}+y^{2} & =e^{-2 n} .
\end{aligned}
$$

Solving this system for $\sin \beta=\frac{y}{e^{-n}}$

$$
\begin{aligned}
-2 y e^{-n} \cosh (\delta n-D)+e^{-2 n} \cosh ^{2}(\delta n-D) & =e^{-2 n}\left(\sinh ^{2}(\delta n-D)-1\right), \\
e^{-n}\left(\cosh ^{2}(\delta n-D)-\sinh ^{1}(\delta n-D)+1\right) & =2 y \cosh (\delta n-D), \\
e^{-n} & =y \cosh (\delta n-D), \\
\frac{y}{e^{-n}} & =\frac{1}{\cosh (\delta n-D)} .
\end{aligned}
$$

Therefore

$$
\sin \beta=\frac{y}{e^{-n}}=\frac{1}{\cosh (\delta n-D)}<\frac{1+e^{2}}{2 e} \frac{1}{\cosh (\delta n-D)}=\sin \alpha .
$$

This means that Figure 3 above is correct and we can use the angle $\alpha$ to give an upper bound for the area $A_{n} \backslash H_{1}$. Using this estimation for the area of $A_{n} \backslash$ $\left(H_{1} \cup H_{2}\right)$, where the dilatation may differ from zero,

$$
\begin{aligned}
\iint_{A_{n} \backslash\left(H_{1} \cup H_{2}\right)} d \sigma & \leq\left[\left(e^{-n+1}\right)^{2}-\left(e^{-n}\right)^{2}\right] \alpha \\
& =e^{-2 n}\left(e^{2}-1\right) \alpha \\
& \leq e^{-2 n}\left(e^{2}-1\right) \frac{\pi}{2} \sin \alpha \\
& \leq e^{-2 n}\left(e^{2}-1\right) \frac{\pi}{2} \frac{\left(e^{2}+1\right) e^{D-1}}{2} e^{-\delta n} \\
& =c e^{-2 n} e^{-\delta n} .
\end{aligned}
$$


Remark 4.3. In general, if $\operatorname{dist}\left(\gamma(t), z_{0}\right) \geq \phi(t)$ for all $t$, then with the same calculation we can show that

$$
\iint_{A_{n} \backslash H_{1}} d \sigma \leq c e^{-2 n} e^{\phi(n)} .
$$

Lemma 4.4. If $x_{0}$ is a deep point, then

$$
I\left(x_{0}\right)=\iint_{D} \frac{\left|\mu(x)-\mu\left(x_{0}\right)\right|}{\left|x-x_{0}\right|^{2}} d \sigma<\infty .
$$

Proof. Using Lemma 4.2 the integral $I\left(x_{0}\right)$ over the Euclidean ball of radius $e^{-N_{0}}$, where $N_{0}$ is an integer bigger than $\frac{1+D}{\delta}$, is

$$
\begin{aligned}
I\left(x_{0}\right) & =\iint \frac{\left|\mu(x)-\mu\left(x_{0}\right)\right|}{\left|x-x_{0}\right|^{2}} d \sigma \\
& \leq \sum_{n=N_{0}}^{\infty} \iint_{A_{n} \backslash\left(H_{1} \cup H_{2}\right)} \frac{\left|\mu(x)-\mu\left(x_{0}\right)\right|}{\left|x-x_{0}\right|^{2}} d \sigma \\
& \leq \sum_{n=N_{0}}^{\infty} \iint_{A_{n} \backslash\left(H_{1} \cup H_{2}\right)} \frac{2}{e^{-2 n}} d \sigma \\
& \leq \sum_{n=N_{0}}^{\infty} \frac{2}{e^{-2 n}} e^{-2 n}\left(e^{2}-1\right) \frac{\pi}{2} \frac{\left(e^{2}+1\right) e^{D-1}}{2} e^{-\delta n} \\
& =\frac{\left(e^{4}-1\right) e^{D-1} \pi}{2} \sum_{n=N_{0}}^{\infty} e^{-\delta n}<\infty .
\end{aligned}
$$

Therefore we have shown that $F$ is differentiable with non-zero derivative at the deep points.

Remark 4.5. In the general case, when $\operatorname{dist}\left(\gamma(t), z_{0}\right) \geq \phi(t)$ for all $t$, then

$$
I\left(x_{0}\right) \leq c \sum_{n=N_{0}}^{\infty} e^{-\phi(n)} .
$$

In particular, if $\phi(t) \geq(1+\epsilon) \ln t$ with some positive $\epsilon$, then

$$
I\left(x_{0}\right) \leq c \sum e^{-\phi(n)} \leq c \sum \frac{1}{n^{1+\epsilon}}<\infty .
$$

\section{HARMONIC FUNCTIONS}

In this section we collect a few facts about harmonic functions and special properties of harmonic functions living on surfaces like the half of the jungle gym, that we will need in the proof of our main theorem.

Theorem 5.1. Suppose $G$ is a geometrically infinite Fuchsian group, $M=\mathbb{B} / G$ has injectivity radius bounded and bounded away from zero, and there is a Green's function on $M$. Let $\phi(t):[1, \infty) \rightarrow[1, \infty)$ be a Lipschitz function satisfying $\lim _{t \rightarrow \infty} \phi(t)=\infty$; then $\operatorname{dim}_{\mathrm{H}}\left(\Lambda_{\phi}\right)=1$. 


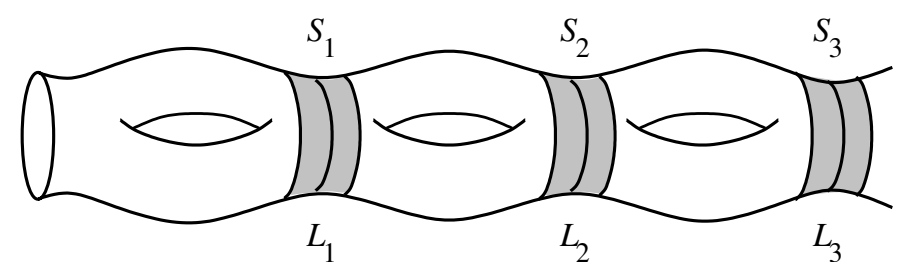

Figure 4. Quasi-cylinder

The proof of this theorem is described in [6] and uses the following lemma from [3].

Lemma 5.2. Suppose $G$ is topologically tame, geometrically infinite, $M=\mathbb{B} / G$ has injectivity radius bounded below by $\epsilon>0$ and Green's function $G(w, z)$ exists on $M$. Then there exists a positive harmonic function $U$ on $M$ such that

$$
\sup _{z \in M}|\nabla U(z)| \leq 1
$$

and $U$ tends to zero in the geometrically finite ends of $M$. If, in addition, $G$ is topologically tame, then for any $a_{0}>0$ there are constants $a_{1}$ and $a_{2}$ so that

$$
\int_{B\left(z, a_{1}\right)}|\nabla U|^{2} d V \geq a_{2}
$$

for every $z$ such that $\operatorname{dist}(z, C(M)) \leq a_{0}$. Moreover, $U(z)$ tends to $+\infty$ in the geometrically infinite ends as $\operatorname{dist}(z, \partial C(M)) \rightarrow \infty$.

Let $M$ be a quasi-cylinder as depicted in Figure 4 . That is, a complete Riemann manifold with compact boundary, and with bounded geometry locally, i.e. each point of $M$ away from the boundary has a ball neighborhood of radius 1 which is a geometrically bounded distortion of the unit ball in Euclidean space. Furthermore, we suppose that $M$ has the following additional properties: let $S_{n}$ denote the hypersurface at distance $n$ from the boundary of $M$ and suppose $S_{n}$ is homologous to $\partial M$ and $\operatorname{diam}\left(S_{n}\right) \leq D$. Suppose there are closed bands $L_{1}, L_{2}, \ldots$ on $M$, so that each band $L_{i}$ contains a unit neighborhood of $S_{i}$ with $\operatorname{vol}\left(L_{i}\right) \leq V$.

Theorem 5.3. Let $M$ be a complete oriented Riemann manifold and let $h$ be a harmonic function on it. Suppose $M_{+}=h^{-1}[0, \infty)$ has a compact boundary and that $h^{-1}[a, b]$ is compact for all $0 \leq a<b<\infty$. Assume that each point of $M_{+}$(away from the boundary) has a ball neighborhood of radius one which is a geometrically bounded distortion of a unit ball in Euclidean space. Then the gradient of $h$ is uniformly bounded on $M_{+}$.

Lemma 5.4. Let $M$ be a quasi-cylinder, and let $S_{n}$ denote the hypersurface at distance $n$ from the boundary of $M$, and suppose $S_{n}$ is homologous to $\partial M$ with $\operatorname{diam}\left(S_{n}\right) \leq D$. Then $M$ admits a non-constant positive harmonic function $h$ so that

$$
\frac{1}{c} \operatorname{dist}(x, \partial M) \leq h(x) \leq c \operatorname{dist}(x, \partial M) .
$$

Theorem 5.3 and Lemma 5.4 are due to D. Sullivan and were published in [14]. 


\section{Proof of the TheOrem AND its COROLlaries}

Now we are ready to prove the main theorem of this paper.

Theorem 6.1. Let $f$ be a quasi-conformal self-map of the jungle gym so that the dilatation of $f$ is compactly supported. Let $F$ be its lifted map to the hyperbolic disc extended to the boundary of the disc. Then $F$ is differentiable with non-zero derivative at the deep points, and the Hausdorff dimension of these points is full.

Proof. In Lemma 4.4 we showed that $I\left(x_{0}\right)$ is finite at every deep point, and so by Lehto's Theorem 4.1 we can conclude that the map $F$ is totally differentiable with non-zero derivative there. Now cut the jungle gym in half. According to Lemma 5.4 there is a positive harmonic function which grows linearly as $x$ goes to infinity on each half. Even though there is no Green's function on this manifold we may still use Theorem 5.1, because the harmonic function on each half of the quasi-cylinder has the properties described in Lemma 5.2. Therefore the Hausdorff dimension of the deep points is 1 . By Theorem 4.1 and Lemma 4.4 the map $F$ is differentiable with non-zero derivative at each of its deep points, so we can conclude that the Hausdorff dimension of those points where $F$ is differentiable with non-zero derivative is 1 .

Remark 6.2. Let $M$ be a hyperbolic manifold so that one of its ends is a quasicylinder, i.e. this end has bounded geometry locally, and if $S_{n}$ denotes the hypersurface at distance $n$ from the beginning of this end, then $S_{n}$ is homologous to $S^{1}$ with $\operatorname{diam}\left(S_{n}\right) \leq D$. Consider a quasi-conformal deformation of this surface $M$ by dilatation with compact support. Lift this deformation to the universal covering space $\mathbb{B}$, extend it to the boundary of $\mathbb{B}$ and call it $F$. Then $F$ is differentiable at the deep points, which have full dimension. Therefore the Hausdorff dimension of the points where $F$ is differentiable with non-zero derivative is also full.

Corollary 6.3. Consider a quasi-symmetric homeomorphism $f$ of the unit circle which conjugates two divergence-type groups with quasi-cylindrical end, like the covering group of the jungle gym. Suppose that the dilatation has compact support. Then $f$ is differentiable with non-zero derivative on a set of dimension 1 , and the image of this set also has dimension 1.

Proof. In Theorem 6.1 we have shown that the set

$$
A=\left\{x: f^{\prime}(x) \text { exists and is non-zero }\right\}
$$

has dimension one. Define the set

$$
A_{M}=\left\{x: \frac{1}{M}<\left|f^{\prime}(x)\right|<M\right\} .
$$

From the definition, $A_{M_{1}} \subset A_{M_{2}}$ whenever $M_{1}<M_{2}$, and $\bigcup_{M} A_{M}=A$. Therefore for all $\epsilon>0$ there exists $A$ so that $\operatorname{dim}_{\mathrm{H}}\left(A_{M}\right) \geq 1-\epsilon$.

First we will show that if $x \in A_{M}$, then there exists a neighborhood $B_{x}$ of $x$ so that $\frac{1}{2 M}\left|B_{x}\right| \leq\left|f\left(B_{x}\right)\right| \leq 2 M\left|B_{x}\right|$. Since $x \in A_{M}$, then

$$
\frac{1}{M} \leq \lim _{y \rightarrow x} \frac{|f(y)-f(x)|}{|y-x|} \leq M .
$$

Therefore if $|y-x|$ is small enough, then $\frac{1}{2 M}|y-x|<|f(y)-f(x)|<2 M|y-x|$. This means we can choose a neighborhood $B_{x}$ around the point $x$ so that

$$
\frac{1}{2 M}\left|B_{x}\right|<\left|f\left(B_{x}\right)\right|<2 M\left|B_{x}\right|
$$


Note that the diameter of this neighborhood depends on $M$ and on the point $x$. To get rid of the dependence on $x$, define the set

$$
A_{M, \delta}=\left\{x \in A_{M}: \forall B_{x} \text { with }\left|B_{x}\right| \leq \delta, \frac{\left|B_{x}\right|}{2 M} \leq\left|f\left(B_{x}\right)\right| \leq 2 M\left|B_{x}\right|\right\} .
$$

Then $A_{M}=\bigcup_{\delta} A_{M, \delta}$ and $A_{M, \delta_{1}} \subset A_{M, \delta_{2}}$ if $\delta_{1}>\delta_{2}$. Therefore, for any small positive epsilon we can find $M$ and $\delta$ so that $\operatorname{dim}_{\mathrm{H}}\left(A_{M, \delta}\right)>1-\epsilon$.

Now we will show that $\operatorname{dim}_{\mathrm{H}}\left(f\left(A_{M, \delta}\right)\right)=\operatorname{dim}_{\mathrm{H}}\left(A_{M, \delta}\right)$. On the one hand, if we take any covering of $A_{M, \delta}$ with balls $\left\{U_{i}\right\}$ of diameter less than $\delta$, then

$$
\frac{\left|U_{i}\right|}{2 M} \leq\left|f\left(U_{i}\right)\right| \leq 2 M\left|U_{i}\right|
$$

and $\left\{f\left(U_{i}\right)\right\}$ gives a cover for $f\left(A_{M, \delta}\right)$. This gives an estimation for the Hausdorff content of $f\left(A_{M, \delta}\right)$ :

$$
\mathcal{H}^{\alpha}\left(f\left(A_{M, \delta}\right)\right) \leq \sum\left|f\left(U_{i}\right)\right|^{\alpha} \leq(2 M)^{\alpha} \sum\left|U_{i}\right|^{\alpha}
$$

for any covering $\left\{U_{i}\right\}$ of the set $A_{M, \delta}$, so

$$
\mathcal{H}^{\alpha}\left(f\left(A_{M, \delta}\right)\right) \leq \mathcal{H}^{\alpha}\left(A_{M, \delta}\right)
$$

and therefore

$$
\operatorname{dim}_{\mathrm{H}}\left(f\left(A_{M, \delta}\right)\right) \leq \operatorname{dim}_{\mathrm{H}}\left(A_{M, \delta}\right) .
$$

On the other hand, take a covering $\left\{V_{i}\right\}$ of the set $f\left(A_{M, \delta}\right)$ so that $\left|V_{i}\right| \leq \frac{\delta}{4 M}$. We may assume that $V_{i} \cap f\left(A_{M, \delta}\right)$ is not empty for any $i$, so there exists a point $x \in f^{-1}\left(V_{i}\right) \cap A_{M, \delta}$. From the definition of the set $A_{M, \delta}$, the point $x$ has a $\delta$ neighborhood $B_{x}$, for which

$$
\frac{\left|B_{x}\right|}{2 M} \leq\left|f\left(B_{x}\right)\right| \leq 2 M\left|B_{x}\right| .
$$

Since $\left|V_{i}\right| \leq \frac{\delta}{4 M}$, the set $V_{i}$ lies inside the image of $B_{x}$, or equivalently $f^{-1}\left(V_{i}\right) \subset B_{x}$, and so by the definition of $A_{M, \delta}$

$$
\frac{\left|f^{-1}\left(V_{i}\right)\right|}{2 M} \leq\left|V_{i}\right| \leq 2 M\left|f^{-1}\left(V_{i}\right)\right| .
$$

The set $A_{M, \delta}$ is covered by $\left\{f^{-1}\left(V_{i}\right)\right\}$, so

$$
\mathcal{H}^{\alpha}\left(A_{M, \delta}\right) \leq \sum\left|f^{-1}\left(V_{i}\right)\right|^{\alpha} \leq(2 M)^{\alpha} \sum\left|V_{i}\right|^{\alpha},
$$

and hence

$$
\mathcal{H}^{\alpha}\left(A_{M, \delta}\right) \leq \mathcal{H}^{\alpha}\left(f\left(A_{M, \delta}\right)\right)
$$

So

$$
\operatorname{dim}_{\mathrm{H}}\left(A_{M, \delta}\right) \leq \operatorname{dim}_{\mathrm{H}}\left(f\left(A_{M, \delta}\right)\right) .
$$

Inequalities (6.1) and (6.2) show that the Hausdorff dimension of $A_{M, \delta}$ is the same as its image under the map $f$. Since the dimension is preserved for every $\delta$ and $M$, $\operatorname{dim}_{\mathrm{H}}(A)=\operatorname{dim}_{\mathrm{H}}(f(A))$.

Corollary 6.4. Under the same conditions as above, we consider a quasi-symmetric homeomorphism $f$ of the unit circle which conjugates two divergence-type groups with quasi-cylindrical end, like the covering group of the jungle gym, such that the dilatation has compact support. We claim that there is no subset $E$ of the circle so that $E$ and $f\left(E^{c}\right)$ both have dimension less than 1 . 


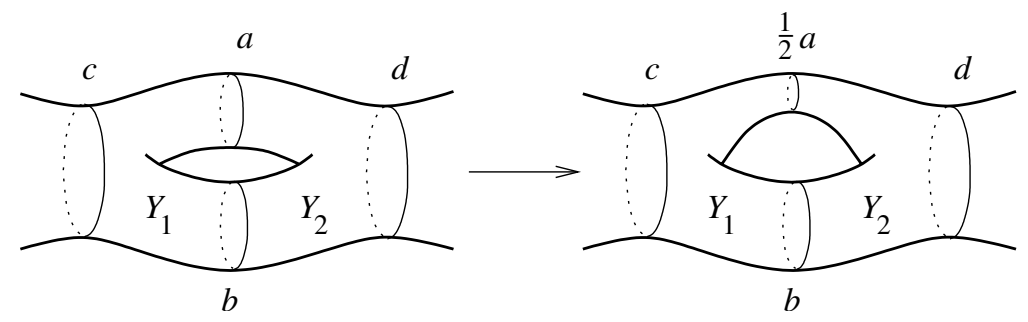

Figure 5. Deformation on the $Y$-pieces

Proof. For the last statement of the corollary assume that $\operatorname{dim}_{\mathrm{H}}(E)=1-\epsilon<1$; then we have to prove that $\operatorname{dim}_{H}\left(f\left(E^{c}\right)\right)=1$. Since the dimension of $E$ is strictly less than 1 but $\operatorname{dim}_{\mathrm{H}}(A)=1$, then

$$
\operatorname{dim}_{\mathrm{H}}(A \cap E) \leq \operatorname{dim}_{\mathrm{H}}(E)=1-\epsilon .
$$

Let us again use the notation $A=\left\{x: f^{\prime}(x)\right.$ exists and is non-zero $\}$. We may write $A$ as the disjoint union of the two sets, $E^{c}=A \backslash E$ and $A \cap E$. Then $\operatorname{dim}_{\mathrm{H}}(A)=$ $\max \left\{\operatorname{dim}_{\mathrm{H}}\left(E^{c}\right), \operatorname{dim}_{\mathrm{H}}(A \cap E)\right\}$, and since $\operatorname{dim}_{\mathrm{H}}(A)=1$ but $\operatorname{dim}_{\mathrm{H}}(A \cap E)=1-\epsilon$, the dimension of $E^{c}$ must be one. The map $f$ preserves the Hausdorff dimension of $F$, as we have shown in the previous corollary; therefore $\operatorname{dim}_{\mathrm{H}}\left(E^{c}\right)=1$ and so does the dimension of $f\left(E^{c}\right)$.

Corollary 6.5. There is a quasi-conformal deformation of a divergence-type group with quasi-cylindrical end on the quotient manifold, so that the limit set might not be a circle, but does have tangents on a set of dimension 1 .

Proof. First, we show that there is a quasi-conformal deformation of the jungle gym so that the limit set is not the circle. Take a $Y$-piece decomposition of the jungle gym. A $Y$-piece is a bordered Riemann surface which is topologically a sphere with three disks removed and in which each of the three boundary components is a hyperbolic geodesic. A $Y$-piece is determined up to isometry by the lengths of the three boundary components. Take two adjacent $Y$-pieces, $Y_{1}$ and $Y_{2}$ with geodesics $(a, b, c)$ and $(a, b, d)$, respectively, as shown in Figure 5. Construct a new surface $S^{\prime}$ so that it is the same as the surface $S$ everywhere except on $Y_{1}$ and $Y_{2}$. Deform these two pieces into $Y_{1}^{\prime}$ and $Y_{2}^{\prime}$ with geodesics $\left(\frac{1}{2} a, b, c\right)$ and $\left(\frac{1}{2} a, b, d\right)$, respectively. The new surface $S^{\prime}$ and the old $S$ are topologically but not conformally equivalent, since the hyperbolic length of the geodesics are not preserved. As it is shown in [5] there is a quasi-conformal mapping $f: S \rightarrow S^{\prime}$. Lifting this map $f$ up to the covering space we get a quasi-conformal map $F$ between the two covering groups $G$ and $G^{\prime}$. We claim that the limit set of $G^{\prime}$ is not the circle.

Outside the disk no deformation happened, so $F$ is conformal on $\mathbb{C} \backslash \mathbb{D}$. If it was a circle, $F$ would be Möbius by the theorem of Tukia [15]. Therefore the surfaces $(\mathbb{C} \backslash \mathbb{D}) / G,(\mathbb{C} \backslash \mathbb{D}) / G^{\prime}$ and $\mathbb{D} / G=S$ would all be conformally equivalent. But then a reflection across the circle $S^{1}$ would give a conformal map between $S$ and $S^{\prime}$, which is not possible.

Consider a deformation of the jungle gym described above. Its dilatation is supported on a compact set of the manifold. The same argument as in Theorem 6.1 shows that the lifted map $F$ is differentiable with a non-zero derivative at the deep 
points. Therefore the quasi-circle has tangents on a set of Hausdorff dimension one.

\section{ACKNOWLEDGEMENT}

I would like to say special thanks to Christopher Bishop for his remarks on the earlier version of this paper.

\section{REFERENCES}

1. S. Agard, A geometric proof of Mostow's rigidity theorem for groups of divergence type, Acta Math. 151 (1983), 231-252. MR0723011 (86b:22017)

2. K. Astala and M. Zinsmeister, Holomorphic families of quasi-Fuchsian groups, Ergodic Theory Dynam. Systems 14 (1994), 207-212. MR.1279468 (95k:30095)

3. C. J. Bishop and P. W. Jones, The law of the iterated logarithm for Kleinian groups, Contemp. Math. 211 (1997), 17-50. MR1476980 (98j:30051)

4. C. J. Bishop and Y. Peres, Hausdorff dimension and Fractal Sets, to appear.

5. C. J. Bishop and T. Steger, Representation-theoretic rigidity in PSL(2, R), Acta Math. 170 (1993), no. 1, 121-149. MR1208564 (94g:22023)

6. Z. Gönye, The Dimension of Escaping Points, Ph.D. thesis, State University of New York at Stony Brook, 2001.

7. O. Lehto, On the differentiability of quasiconformal mapping with prescribed complex dilatation, Ann. Acad. Sci. Fenn. Ser. A I 275 (1960), 28. MR0125963 (23:A3260)

8. Conformal Mappings and Teichmüller Spaces, Israel Institute of Technology, Haifa, Israel, 1973.

9. — Univalent Functions and Teichmüller Spaces, Springer-Verlag, New York, 1987. MR 0867407 (88f:30073)

10. O. Lehto and K. I. Virtanen, Quasiconformal Mappings in the Plane, Springer-Verlag, New York, 1973. MR0344463(49:9202)

11. G. D. Mostow, Quasiconformal mappings in n-space and the rigidity of hyperbolic space forms, Inst. Hautes Études Sci. Publ. Math. 34 (1968), 53-104. MR.0236383 (38:4679)

12. Strong rigidity of locally symmetric spaces, Ann. of Math. Stud., vol. 78, Princeton University Press, Princeton, 1973. MR0385004 (52:5874)

13. P. J. Nicholls, The Ergodic Theory of Discrete Groups, Cambridge University Press, Cambridge, 1989. MR1041575 (91i:58104)

14. D. Sullivan, Growth of positive harmonic functions and Kleinian group limit sets of zero planar measure and Hausdorff dimension two, Geometry Symposium 504 (1980), 127-144. MR:0655423 (83h:53054)

15. P. Tukia, Differentiability and rigidity of Möbius groups, Invent. Math. 82 (1985), 557-578. MR0811551 (87f:30058)

Department of Mathematics, Polytechnic University, Brooklyn, New York 11201

E-mail address: zgonye@poly.edu 